\title{
On the asymmetry of the distribution of observable comets induced by a star passage through the Oort cloud
}

\author{
P. A. Dybczyński`
}

\begin{abstract}
Astronomical Observatory of the A. Mickiewicz University, ul. Słoneczna 36, 60-286 Poznań, Poland
Received 5 October 2001 / Accepted 28 November 2001

Abstract. Single passage of a star through the Oort cloud yields anisotropic distribution of observable long period comets. This conclusion emerges from our extensive Monte Carlo simulations of the directional distribution of longperiod comets induced by a stellar passage through the Oort cloud. For a broad range of simulation parameters we obtained the asymmetric distribution of comets as result of a single stellar passage. A direct result of our simulation is a guide-line for studies of the anisotropies in the observed long-period comet sample and searching for fingerprints of a recent stellar passage. We obtained an isotropic distribution, as simulated by Weissman (1996) only for a very peculiar choice of input parameters. The accuracy of our calculations was verified by consistency of the impulse approximation and direct orbit integration results.
\end{abstract}

Key words. comets: general - Oort cloud - solar system: general

\section{Introduction}

The problem of the existence and explanation of the anisotropies in the directional distribution of the observed long-period comet sample plays an important role in the studies of the origin of comets. Before 1950 (i.e. when an interstellar cometary origin hypothesis dominated) many attempts were made to find any anisotropies correlated with the solar motion. After the wide acceptance of the Oort cloud hypothesis (Oort 1950) the existence of these asymmetries was reexamined by many authors and new explanations were proposed. According to Biermann et al. (1983) the observed weak concentrations of the perihelion points of the long period comets can be explained as a result of some recent stellar passages through or near the cometary cloud. However Weissman (1996) (hereafter W96) investigated the results of the direct passage of a star through the Oort cloud of comets and concluded that the resulting population of the observed long-period comets is isotropic. As a consequence, some authors tried to find other explanations (see for example Matese et al. 1999).

We performed an extensive set of Monte Carlo simulations in order to again study the results of a single stellar passage through or near the Oort cometary cloud in more detail (Sect. 1). Section 3 is devoted to discussion of a special, singular case in our calculations. The numerical accuracy of our computations was verified by calculation of the same cases in the impulse approximation and by direct numerical integration (Sect. 4).

\footnotetext{
* e-mail: dybol@amu.edu.pl
}

\section{The simulations}

Using the original Monte Carlo simulation code we calculated the effects of stellar passages for wide ranges of the parameters. The perturber mass varied from 0.5 up to 5 solar masses, the minimum heliocentric distance from 10000 to $100000 \mathrm{AU}$ and the stellar perturber velocity from $5 \mathrm{~km} \mathrm{~s}^{-1}$ to $50 \mathrm{~km} \mathrm{~s}^{-1}$. As a model of the Oort cloud we used the spherically symmetric cloud with Duncan et al. (1987) distributions of the cometary semimajor axis and the eccentricity and assumed a uniform distribution of the mean anomaly of comets. Any errors introduced by numerical reconstruction of the semimajor axis and eccentricity distributions from the plots presented in Duncan et al. (1987) are of negligible importance for our results. We used a classical impulse approximation and the inner boundary $(I B)$ of the simulated cloud was $50 \mathrm{AU}$ whereas the outer boundary $(O B)$ value was $2 \times 10^{5} \mathrm{AU}$. We assumed the observability limit $(O L)$ to be equal to $10 \mathrm{AU}$.

We illustrate here some of the results on the basis of one example: the passage of one solar mass perturber within $10000 \mathrm{AU}$ from the Sun, moving at a velocity of $30 \mathrm{~km} \mathrm{~s}^{-1}$. One way to search for anisotropic results of such a passage (used among others by W96) is to plot the rectangular positions of comets made observable (i.e. with perihelion distance decreased below the observability limit, $q<10 \mathrm{AU}$ ) by the perturbation from the passing star. The positions of all those comets were recorded at the moment of the closest stellar position. The cometary cloud simulated population was in this case $2 \times 10^{7}$. We present this plot in Fig. 1 in two different projections, onto the $X Y$ plane defined by the stellar path at $x=10000 \mathrm{AU}$ and the Sun at the origin (Fig. 1a) and onto the plane $Y Z$, 

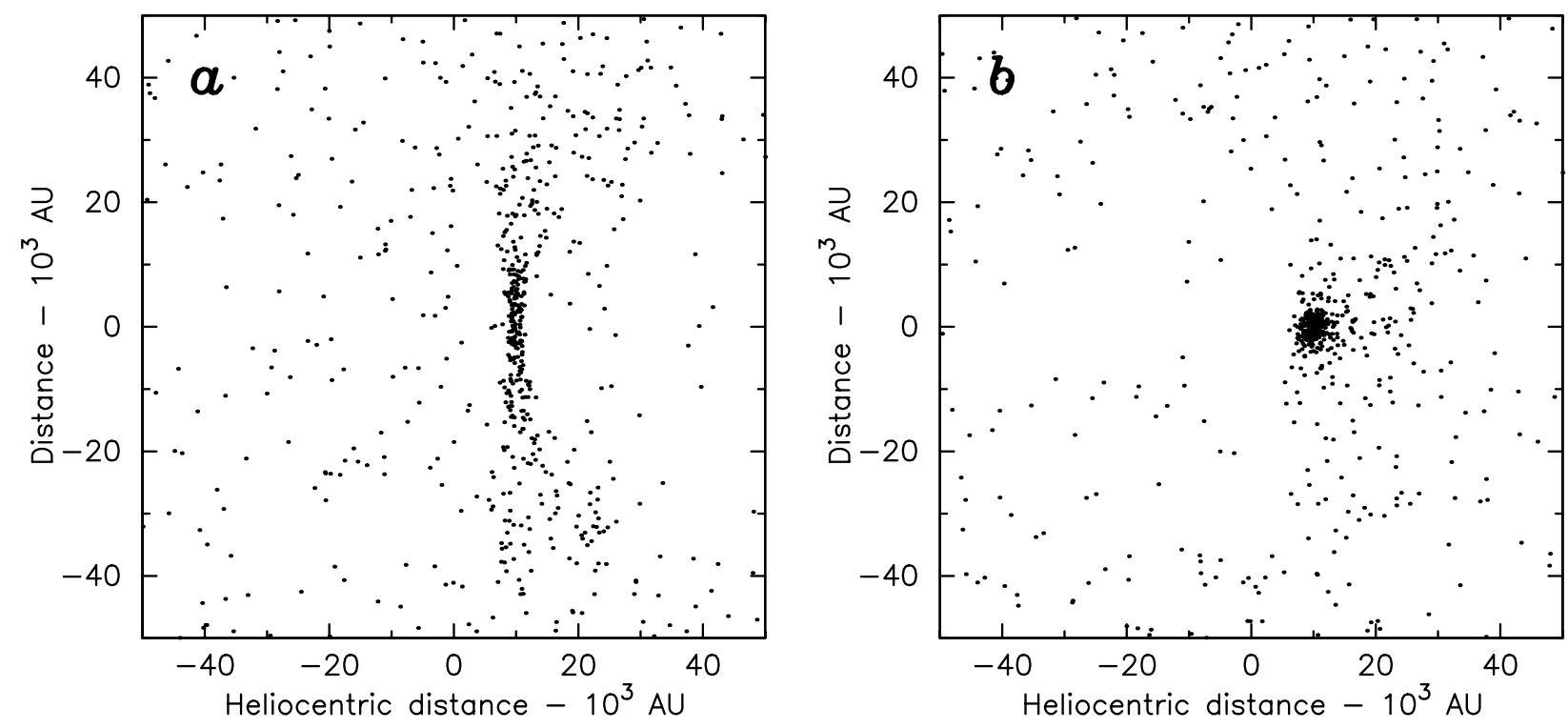

Fig. 1. The positions of comets perturbed to $q<10 \mathrm{AU}$, obtained from our simulation for $\mathrm{IB}=50 \mathrm{AU}$ and recorded at the moment of the minimum Sun-star distance.

perpendicular to the previous one (Fig. 1b). The coordinate system is oriented in such a manner that the stellar straight line path is parallel to the $\mathrm{O} X$ axis and the $X Y$ plane contains both the stellar path and the Sun.

Inspection of Fig. 1a reveals a marked anisotropy of distribution of the perturbed comets, which are potentially observable because of a small $q$. Note that Fig. 1a is the projection of all comets from the cloud so it also includes those lying high above and deep below the $X Y$ plane. The asymmetry would be more pronounced in a cross-section of the cloud with only comets having, say, $|z| \leq 2000$ AU plotted. Then the left two-third of the plot would be almost empty! The explanation comes from Fig. 1b, which presents the same distribution as Fig. 1a, but projected onto the $Y Z$ plane.

While such asymmetries are clearly visible in Figs. 1a,b, the use of the rectangular coordinates of the comets at the moment of the stellar passage seems not to be the best choice. We prefer (and present here in Fig. 2) the distribution of the perihelion directions (as points on the celestial sphere) for the observable subsample of comets obtained in the same manner as in Fig. 1, but enlarged forty times in number to obtain a more readable plot.

In Fig. 2 one can find stellar path parameters (upper left corner), as well as simulated cloud parameters (at the bottom). A strong concentration of perihelion directions along the stellar path near its anti-perihelion is clearly visible as well as the empty region surrounding its perihelion. This picture can be far more impressive if we add here the time-dependence. By restricting the subsample to the comets arriving at the perihelion during the first 500000 years after the stellar passage we obtain the plot presented in Fig. 3.

The anisotropy in the distribution of the perihelion directions is clearly visible here. It should be stressed that the presented example is by no means favorable for inducing such an asymmetry - on the contrary, in every case of greater Sun-star minimum distance the resulting asymmetry is remarkably stronger. Additionally we tested several cases with a smaller inner boundary value - the anisotropy arises for all values of $I B>10.5$.

The consequences of that fact are very important since it becomes feasible to search for recent perturbers among stars in the solar vicinity based on the observed longperiod comet orbit distribution asymmetries.

\section{The isotropic case}

The setup of our calculations is in many respects the same as in W96 but we do obtain the isotropic distribution in one special case when the inner boundary $I B$ value is the same as the observability threshold value, namely $10 \mathrm{AU}$. After the search through the parameter space, we found that an isotropic distribution may be obtained only in this case. It is really surprising, but even for $I B=10.5 \mathrm{AU}$ the plots obtained are substantially anisotropic. Using the inner boundary value equal to the observability limit we have come across a very strange effect: an arbitrarily small perturbation can make an orbit observable, the proverbial beating of a butterfly's wings may be sufficient! Moreover, such a low value of the $I B$ is in conflict with standard Oort cloud model: typically we call a comet a member of the cloud if its orbit is safe from planetary perturbations which is not the true for $I B=10 \mathrm{AU}$.

The results produced by our code for this rather special case are displayed in Fig. 4 and a comparison of this plot with Figs. 2c,d of W96 reveals no differences.

It is also interesting to compare total statistics of (i) the number of comets ejected to the space on hyperbolic orbits, (ii) the number of comets perturbed to aphelia greater than $200000 \mathrm{AU}$ (called lost) and (iii) the number 


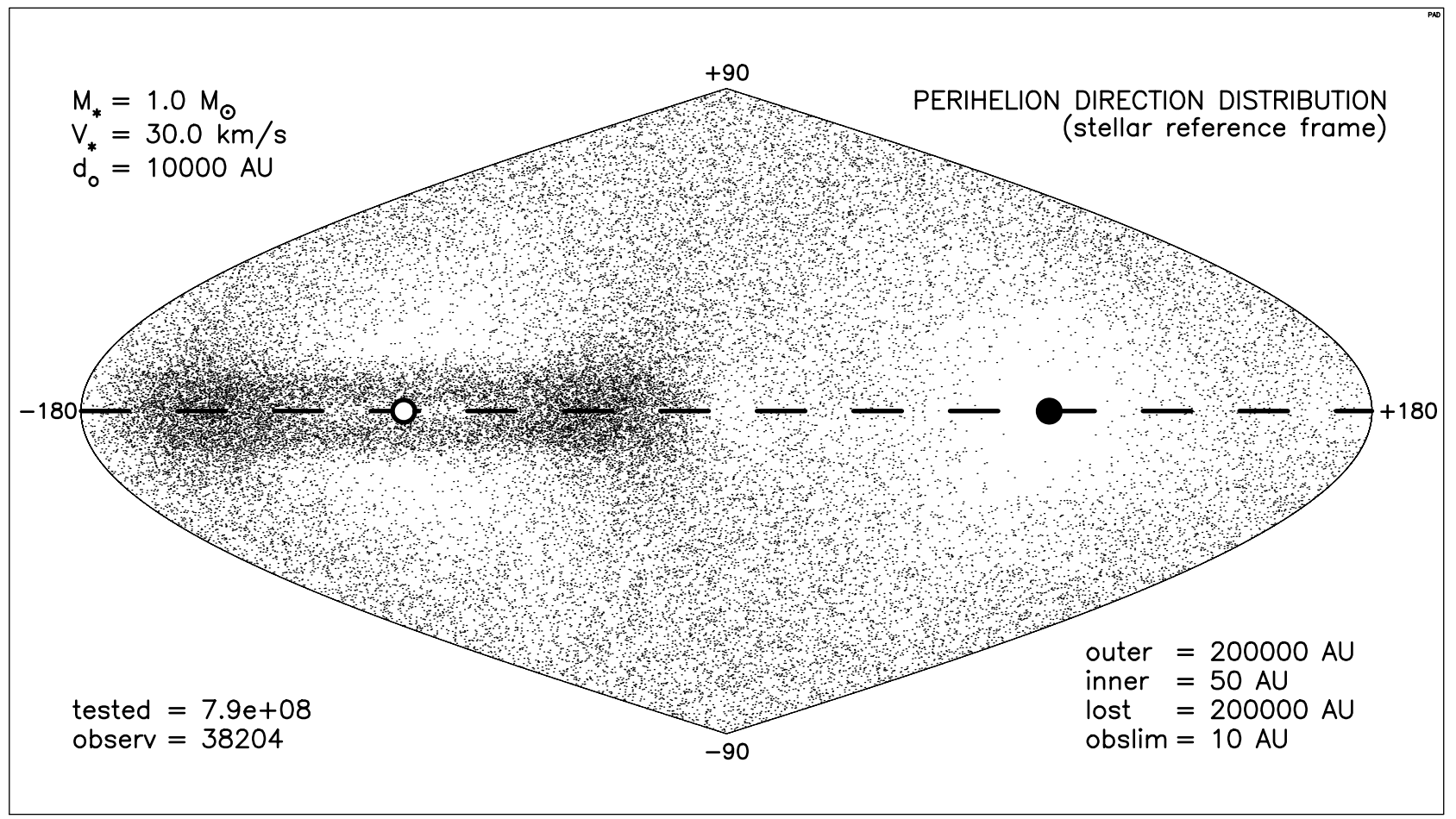

Fig. 2. Perihelion points direction distribution for the observable subsample of comets, presented in the equal-area projection of the celestial sphere. The dashed line is the stellar path projection with an empty circle at star perihelion and full circle at its anti-perihelion.

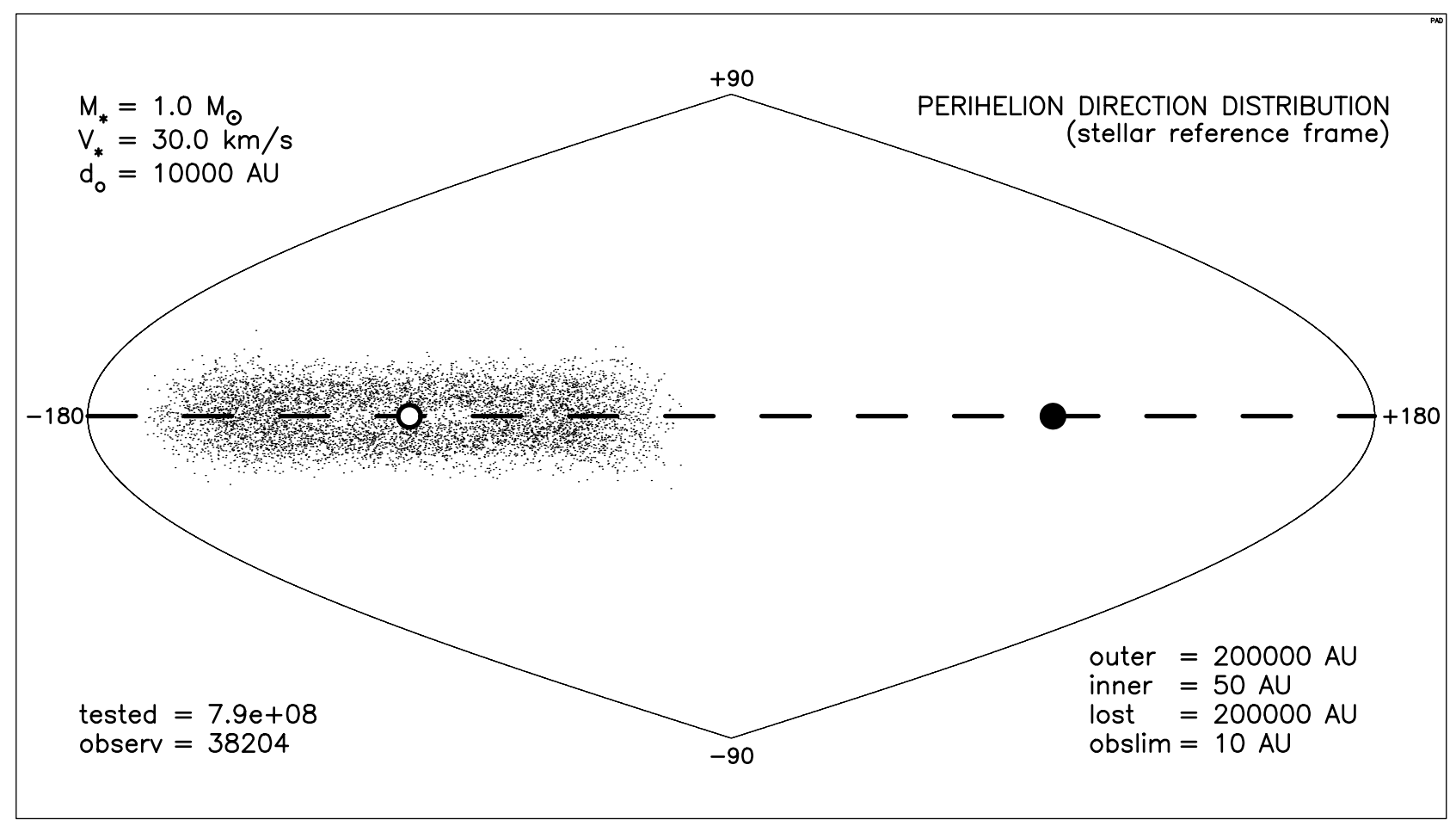

Fig. 3. Same as in Fig. 2, but limited to comets, which arrive at perihelion no later than 500000 years after the stellar passage.

of comets perturbed to perihelion distances less than $10 \mathrm{AU}$ (called observable) for different cases mentioned above. The first two numbers do not strongly depend on $I B$ - both results, for $I B=10 \mathrm{AU}$ and $I B=50 \mathrm{AU}$ are almost the same. This is not the case for the number of observable comets produced by the stellar passage as it strongly depends on $I B$.

In Table 1 we present values of the probabilities of three different end-states mentioned above obtained from different simulations. The last row contains the results 

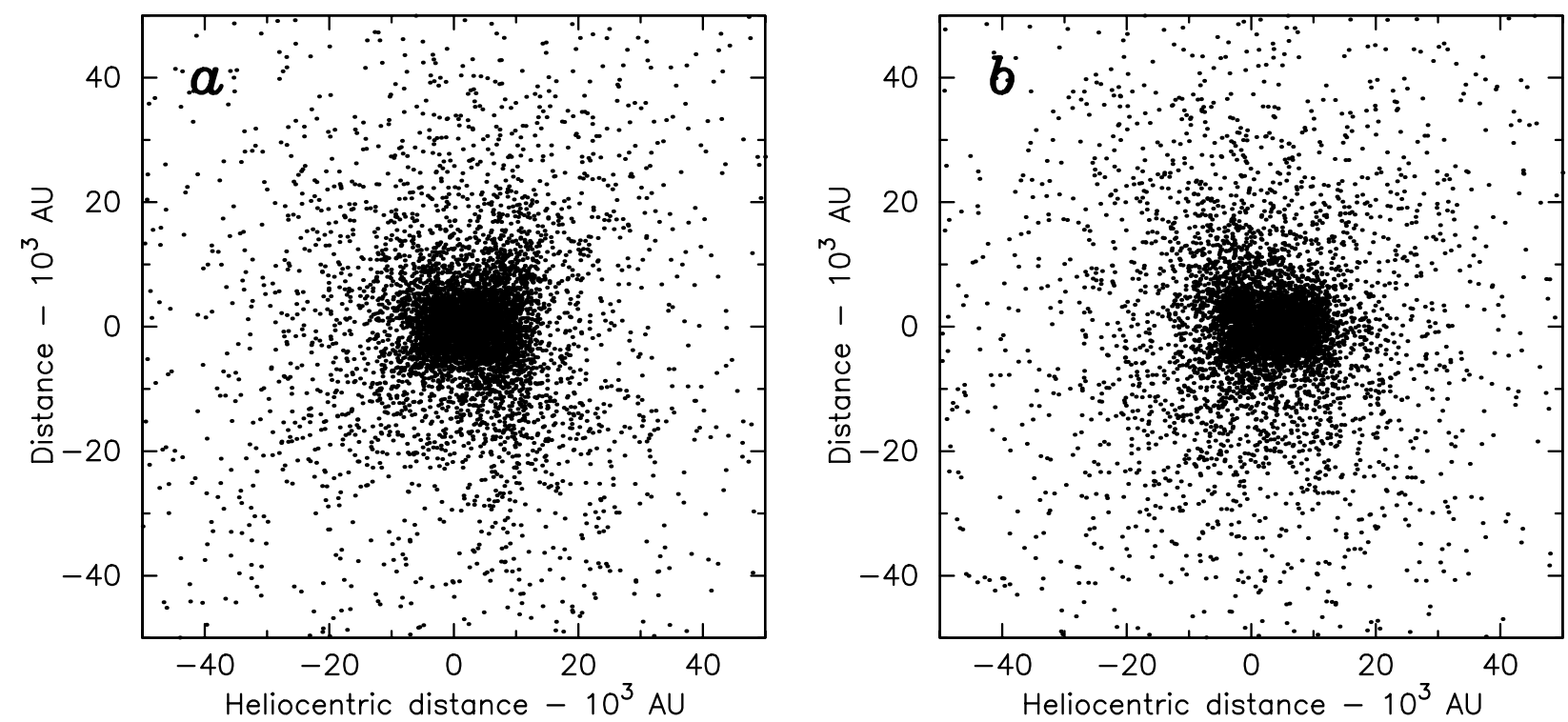

Fig. 4. The same as in Fig. 1 but for $I B=10$ AU. Note that this is a rather peculiar and hardly acceptable value of the parameter $I B$ (see text for explanation).

Table 1. The probabilities of various "end states" for comets from the simulated Oort cloud as a result of a stellar passage described in the text.

\begin{tabular}{lccc}
\hline & Hyperbolic ejection & Being lost (see text) & Becoming observable \\
\hline W96, $I B=50$ (?) AU, imp. approx. & $8.5 \times 10^{-5}$ & $2.3 \times 10^{-4}$ & $1.1 \times 10^{-4}$ \\
This paper, $I B=10 \mathrm{AU}$, imp. approx. & $(8.96 \pm 0.32) \times 10^{-5}$ & $(1.00 \pm 0.01) \times 10^{-3}$ & $(3.65 \pm 0.06) \times 10^{-4}$ \\
This paper, $I B=50 \mathrm{AU}$, imp. approx. & $(8.94 \pm 0.10) \times 10^{-5}$ & $(1.02 \pm 0.003) \times 10^{-3}$ & $(4.83 \pm 0.07) \times 10^{-5}$ \\
This paper, $I B=50 \mathrm{AU}$, num. integr. & $(8.93 \pm 0.08) \times 10^{-5}$ & $(1.02 \pm 0.003) \times 10^{-3}$ & $(4.83 \pm 0.06) \times 10^{-5}$
\end{tabular}

obtained with the exact numerical integration discussed in the following section. Marked differences among our and W96 calculations arise especially in the probability of being lost or becoming observable. No simple explanation is offered at this point.

\section{Validity of the classical impulse approximation}

Finally we decided to repeat the whole simulation by means of the exact numerical integration of a large number of separate three body problems (Sun-star-comet). The aim of such a calculation was to verify our results and discuss the validity of the classical impulse approximation in this case. There are two potential weak points we took into account: the classical impulse approximation might not work well when arbitrarily small comet-star distance is allowed as it is shown in Dybczyński (1994) and, what is more important here, this method sometimes fails when dealing with comets from the inner core, as is shown in Eggers \& Woolfson (1996). The reason for this is that those comets move too fast - the impulse approximation assumes that a comet is at rest during the whole stellar passage.
We developed a completely new computer code, based on numerical integration using D2RKD 7 routine (Fox 1984). Only the random orbit generators were the same. The results showed that both objections to using the classical impulse approximation are not important here: the distribution of perihelion passage directions (see Fig. 5) as well as all probabilities (see Table 1, last row) were exactly the same as in the case of usage of the classical impulse approximation. It appears that whereas for individual, critical cometary orbits those objections are fully justifiable, for statistical description of the simulated comet sample they are of no consequence, at least for the characteristics presented here.

\section{Conclusions}

We studied effects of a stellar passage through the Oort cloud on the origin of the observable long period comets and anisotropy of their distribution. We simulated the spherically symmetric cloud of comets according to the distributions found by Duncan et al. (1987) using a samples of $10^{7}-10^{9}$ comets. The effects of a stellar passage were calculated by means of the classical impulse 


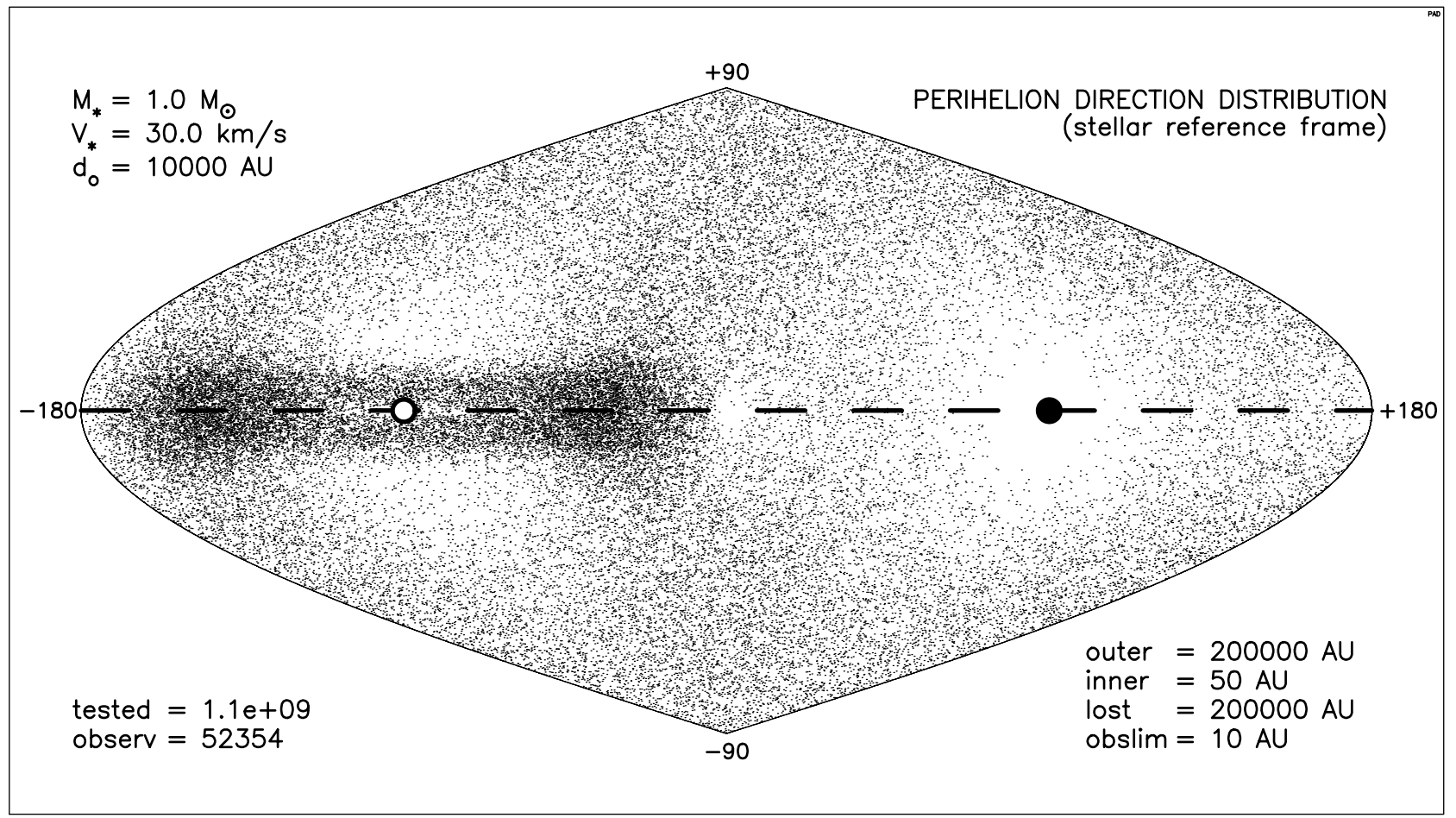

Fig. 5. The same as in Fig. 3 but obtained with the exact numerical integration of separate three body (Sun - star - comet) problems constituting the simulation.

approximation. The validity of the impulse approximation was verified in our case by direct numerical integration of the equations of motion.

In all cases with the inner boundary greater than the observability limit, $I B>O L$ and for a range of other parameters, our simulations yielded markedly anisotropic distribution of the observable comets sample. This has important consequences because the observed concentrations of the perihelia directions of the observed sample of the long-period comets may be analyzed as fingerprints of the recent stellar perturbation. Alternatively we could proceed in the reverse direction and search for candidates for recent perturbers among stars in the solar neighbourhood, simulate their effects and compare with the observed sample of the long period comets. This work is in progress.

The possibility of identifying the recent stellar perturber is also important from the point of view of estimating the current flux of long-period comets delivered from the Oort cloud reservoir by all possible perturbations. While galactic tides do not introduce signifficant variations of the new long-period comets flux, stellar perturbations can increase it by a factor of five or even more (Heisler 1990) during the finite time interval. The duration of this increased flux period depends on the parameters of the stellar passage and typically extends for 0.5 to $3 \mathrm{My}$. When verifying various ideas of the cometary origin it is essential to estimate the steady state flux of new comets and to judge whether we are now experiencing the increased flux or not.
We do not observe an isotropic distribution of the observable long-period comets other than for a rather peculiar choice of parameters, namely for $I B=O L$. In such a case from the outset many comets were barely observable and any infinitesimal push by the stellar perturbation suffices to make a comet observable. Such a situation does not appear realistic, as would call for a large body of comets with isotropic orbit distributions at the giant planet distances. Observations indicate a highly flattened outer Solar System and the Kuiper belt, instead. In this special case our results resemble those of W96 rather closely, as may be appreciated by comparison of his Figs. 2c and 2d with our Fig. 4.

Acknowledgements. The author is indebted to Professor Aleksander Schwarzenberg-Czerny for valuable comments on the first version of this article. Presented computations were partially aided by the KBN grant $8 \mathrm{~T} 12 \mathrm{E} 02820$.

\section{References}

Biermann, L., Huebner, W. F., \& Lüst, R. 1983, Proc. Natl. Acad. Sci. USA, 80, 5151

Duncan, M., Quinn, T., \& Tremaine, S. 1987, AJ, 94, 1330

Dybczyński, P. A. 1994, Cel. Mech. Dyn. Astron., 58, 139

Eggers, S., \& Woolfson, M. M. 1996, MNRAS, 282, 13

Fox, K. 1984, Cel. Mech., 33, 127

Heisler, J. 1990, Icarus, 88, 104

Matese, J. J., Whitman, P. G., \& Whitmire, D. P. 1999, Icarus, 141,354

Oort, J. H. 1950, Bull. Astron. Inst. Nether., 11, 91

Weissman, P. R. 1996, Earth Moon Planets, 72, 25 